\title{
GRN: Gated Relation Network to Enhance Convolutional Neural Network for Named Entity Recognition*
}

\author{
Hui Chen, ${ }^{\dagger}$ Zijia Lin, ${ }^{\ddagger}$ Guiguang Ding, ${ }^{\dagger}$ Jianguang Lou, ${ }^{\ddagger}$ Yusen Zhang, ${ }^{\S}$ Borje Karlsson ${ }^{\ddagger}$ \\ ${ }^{\dagger}$ Beijing National Research Center for Information Science and Technology (BNRist) \\ School of Software, Tsinghua University, Beijing, China \\ ${ }^{\S}$ School of Computer Science, Beijing Institute of Technology, Beijing, China \\ ${ }^{\ddagger}$ Microsoft Research, Beijing, China \\ \{jichenhui2012, yusenzhang95\}@gmail.com,dinggg@tsinghua.edu.cn, \{zijlin, jlou, borje.karlsson\}@ microsoft.com
}

\begin{abstract}
The dominant approaches for named entity recognition (NER) mostly adopt complex recurrent neural networks (RNN), e.g., long-short-term-memory (LSTM). However, RNNs are limited by their recurrent nature in terms of computational efficiency. In contrast, convolutional neural networks $(\mathrm{CNN})$ can fully exploit the GPU parallelism with their feedforward architectures. However, little attention has been paid to performing NER with CNNs, mainly owing to their difficulties in capturing the long-term context information in a sequence. In this paper, we propose a simple but effective CNN-based network for NER, i.e., gated relation network (GRN), which is more capable than common CNNs in capturing long-term context. Specifically, in GRN we firstly employ CNNs to explore the local context features of each word. Then we model the relations between words and use them as gates to fuse local context features into global ones for predicting labels. Without using recurrent layers that process a sentence in a sequential manner, our GRN allows computations to be performed in parallel across the entire sentence. Experiments on two benchmark NER datasets (i.e., CoNLL2003 and Ontonotes 5.0) show that, our proposed GRN can achieve state-of-the-art performance with or without external knowledge. It also enjoys lower time costs to train and test.
\end{abstract}

\section{Introduction}

Named Entity Recognition (NER) is one of the fundamental tasks in natural language processing (NLP). It is designed to locate a word or a phrase that references a specific entity, like person, organization, location, etc., within a text sentence. It plays a critical role in NLP systems for question answering, information retrieval, relation extraction, etc. And thus many efforts have been dedicated to the field.

Traditional NER systems mostly adopt machine learning models, such as Hidden Markov Model (HMM) (Bikel et al. 1997) and Conditional Random Field (CRF) (McCallum and Li 2003). Although these systems can achieve high performance, they heavily rely on hand-crafted features or taskspecific resources (Ma and Hovy 2016), which are expensive to obtain and hard to adapt to other domains or languages.

With the development of deep learning, recurrent neural network (RNN) along with its variants have brought great

\footnotetext{
*Work was done during an internship at Microsoft Research. Copyright (c) 2019, Association for the Advancement of Artificial Intelligence (www.aaai.org). All rights reserved.
}

success to the NLP fields, including machine translation, syntactic parsing, relation extraction, etc. RNNs have been proved to be powerful in learning from basic components of text sentences, like words and characters (Tran, MacKinlay, and Yepes 2017). Therefore, currently the vast majority of state-of-the-art NER systems are based on RNNs, especially long-short-term-memory (LSTM) (Hochreiter and Schmidhuber 1997) and its variant Bi-directional LSTM (BiLSTM). For example, Huang et al. (2015) firstly used a BiLSTM to enhance words' context information for NER and demonstrated its effectiveness.

However, RNNs process the sentence in a sequential manner, because they typically factor the computation along the positions of the input sequence. As a result, the computation at the current time step is highly dependent on those at previous time steps. This inherently sequential nature of RNNs precludes them from fully exploiting the GPU parallelism on training examples, and thus can lead to higher time costs to train and test.

Unlike RNNs, convolutional neural network (CNN) can deal with all words in a feed-forward fashion, rather than composing representations incrementally over each word in a sentence. This property enables CNNs to well exploit the GPU parallelism. But in the NER community, little attention has been paid to performing NER with CNNs. It is mainly due to the fact that CNNs have the capacity of capturing local context information but they are not as powerful as LSTMs in capturing the long-term context information. Although the receptive field of CNNs can be expanded by means of stacking multiple convolutional layers or using dilated convolutional layers, the global context capturing issue still remains, especially for variant-sized text sentences, which hinders CNNs obtaining comparable performance as LSTMs for NER.

In this paper, we propose a CNN-based network for NER, i.e., Gated Relation Network (GRN), which is more powerful than common CNNs for capturing long-term context information. Different from RNNs that capture the long-term dependencies in a recurrent component, our proposed GRN aims to capture the dependencies within a sentence by modelling the relations between any two words. Modelling word relations permits GRN to compose global context features without regard to the limited receptive fields of CNNs, enabling it to capture the global context information. This al- 
lows GRN to reach comparable performance in NER versus LSTM-based models. Moreover, without any recurrent layers, GRN can be trained by feeding all words concurrently into the neural network at one time, which can generally improve efficiency in training and test.

Specifically, the proposed GRN is customized into 4 layers, i.e., the representation layer, the context layer, the relation layer and the CRF layer. In the representation layer, like previous works, a word embedding vector and a characterlevel embedding vector extracted by a CNN are used as word features. In the context layer, CNNs with various kernel sizes are employed to transform the word features from the embedding space to the latent space. The various CNNs can capture the local context information at different scales for each word. Then, the relation layer is built on top of the context layer, which aims to compose a global context feature for a word via modelling its relations with all words in the sentence. Finally, we adopt a CRF layer as the loss function to train GRN in an end-to-end manner.

To verify the effectiveness of the proposed GRN, we conduct extensive experiments on two benchmark NER datasets, i.e., CoNLL-2003 English NER and OntoNotes 5.0. Experimental results indicate that GRN can achieve state-of-the-art performance on both CoNLL$2003\left(\mathrm{~F}_{1}=91.44\right.$ without external knowledge and $\mathrm{F}_{1}=92.34$ with ELMo (Peters et al. 2018) simply incorporated) and Ontonotes $5.0\left(\mathrm{~F}_{1}=87.67\right)$, meaning that using GRN, CNNbased models can compete with LSTM-based ones for NER. Moreover, GRN can also enjoy lower time costs for training and test, compared to the most basic LSTM-based model.

Our contributions are summarized as follows.

- We propose a CNN-based network, i.e., gated relation network (GRN) for NER. GRN is a simple but effective CNN-based architecture with a more powerful capacity of capturing the global context information in a sequence than common CNNs.

- We propose an effective approach for GRN to model the relations between words, and then use them as gates to fuse local context features into global ones for incorporating long-term context information.

- With extensive experiments, we demonstrate that the proposed CNN-based GRN can achieve state-of-the-art NER performance comparable to LSTM-based models, while enjoying lower training and test time costs.

\section{Related Work}

Traditional NER systems mostly rely on hand-crafted features and task-specific knowledge. In recent years, deep neural networks have shown remarkable success in the NER task, as they are powerful in capturing the syntactic dependencies and semantic information for a sentence. They can also be trained in an end-to-end manner without involving subtle hand-crafted features, thus relieving the efforts of feature engineering.

LSTM-based NER System. Currently, most state-of-theart NER systems employ LSTM to extract the context information for each word. Huang et al. (2015) firstly proposed to apply a BiLSTM for NER and achieved a great success.
Later Ma and Hovy (2016) and Chiu and Nichols (2016) introduced character-level representation to enhance the feature representation for each word and gained further performance improvement. MacKinlay et al. (2017) proposed to stack BiLSTMs with residual connections between different layers of BiLSTM to integrate low-level and high-level features. Liu et al. (2018) further proposed to enhance the NER model with a task-aware language model.

Though effective, the inherently recurrent nature of RNNs/LSTMs makes them hard to be trained with full parallelization. And thus here we propose a CNN-based network, i.e., gated relation network (GRN), to dispense with the recurrence issue. And we show that the proposed GRN can obtain comparable performance as those state-of-theart LSTM-based NER models while enjoying lower training and test time costs.

Leveraging External Knowledge. It has been shown that external knowledge can greatly benefit NER models. External knowledge can be obtained by means of external vocabulary resources or pretrained knowledge representation, etc. Chiu and Nichols (2016) obtained $\mathrm{F}_{1}=91.62 \%$ on CoNLL2003 by integrating gazetteers. Peters et al. (2017) adopted a character-level language model pretrained on a large external corpus and gained substantial performance improvement. More recently, Peters et al. (2018) proposed ELMo, a deep language model trained with billions of words, to generate dynamic contextual word features, and gained the latest state-of-the-art performance on CoNLL-2003 by incorporating it into a BiLSTM-based model. Our proposed GRN can also incorporate external knowledge. Specifically, experiments show that, with ELMo incorporated, GRN can obtain even slightly superior performance on the same dataset.

Non-Recurrent Networks in NLP. The efficiency issue of RNNs has started to attract attention from the NLP community. Several effective models have also been proposed to replace RNNs. Gehring et al. (2017) proposed a convolutional sequence-to-sequence model and achieved significant improvement in both performance and training speed. Vaswani et al. (2017) used a self-attention mechanism for machine translation and obtained remarkable translation performance. Our proposed GRN is also a trial to investigate whether CNNs can get comparable NER performances as LSTM-based models with lower time costs for training and test. And different from (Gehring et al. 2017; Vaswani et al. 2017), we do not adopt the attention mechanism here, though GRN is a general model and can be customized into the attention mechanism easily.

Iterated dilated CNN (ID-CNN), proposed by Strubell et al. (2017), also aims to improve the parallelization of NER models by using CNNs, sharing similar ideas to ours. However, although ID-CNN uses dilated CNNs and stacks layers of them, its capacity of modelling the global context information for a variant-sized sentence is still limited, and thus its performance is substantially inferior to those of the state-of-the-art LSTM-based models. In contrast, our proposed GRN can enhance the CNNs with much more capacity to capture global context information, which is mainly attributed to that the relation modelling approach in GRN allows to model long-term dependencies between words with- 
out regard to the limited receptive fields of CNNs. And thus GRN can achieve significantly superior performance than ID-CNN.

\section{Proposed Model}

In this section, we discuss the overall NER system utilizing the proposed GRN in detail. To ease the explanation, we organize our system with 4 specific layers, i.e., the representation layer, the context layer, the relation layer and the CRF layer. We will elaborate on these layers from bottom to top in the following subsections.

\section{Representation Layer}

Representation layer aims to provide informative features for the upper layers. The quality of features has great impacts on the system's performance. Traditionally, features are hand-crafted obeying some elaborative rules that may not be applicable to other domains. Therefore, currently many state-of-the-art approaches tend to employ deep neural networks for automatic feature engineering.

As previous works like (Ye and Ling 2018), the representation layer in GRN is comprised of only word-level features and character-level features. In this paper, we use pretrained static word embeddings, i.e., GloVe ${ }^{1}$ (Pennington, Socher, and Manning 2014), as the initialized word-level feature. And during training, they will be fine-tuned. Here we denote the input sentence $s$ as $s=\left\{s_{1}, s_{2}, \ldots, s_{T}\right\}$, where $s_{i}$ with $i=1,2, \ldots, T$ denotes the $i$ th word in the sentence, and $T$ is the length of the sentence. We also use $\boldsymbol{y}=\left\{y_{1}, y_{2}, \ldots, y_{T}\right\}$ to denote the corresponding entity labels for all words, i.e., $y_{i}$ corresponding to $s_{i}$. With each word $s_{i}$ represented as a one-hot vector, its word-level feature $w_{i}$ is extracted as below:

$$
w_{i}=E\left(s_{i}\right)
$$

where $E$ is the word embedding dictionary, initialized by the GloVe embeddings and fine-tuned during training.

Furthermore, we augment the word representation with the character-level feature, which can contribute to ease the out-of-vocabulary problem (Rei, Crichton, and Pyysalo 2016). Same as (Ma and Hovy 2016), here we adopt a CNN to extract the character-level feature for each word $s_{i}$, as illustrated in Figure 1.

Specifically, the $j$-th character in the word $s_{i}$ containing $n$ characters is firstly represented as an embedding vector $c_{j}^{i}$ in a similar manner as Eq. 1, except that the character embedding dictionary is initialized randomly. Then we use a convolutional layer to involve the information of neighboring characters for each character, which is critical to exploiting $\mathrm{n}$-gram features. Finally, we perform a max-over-time pooling operation to reduce the convolution results into a single embedding vector $c_{i}$ :

$$
\begin{aligned}
\bar{c}_{j}^{i} & =\operatorname{conv}\left(\left[c_{j-k / 2}^{i}, \ldots, c_{j}^{i}, \ldots, c_{j+k / 2}^{i}\right]\right) \\
c_{i} & =\operatorname{pooling}\left(\left[\bar{c}_{0}^{i}, \ldots, \bar{c}_{j}^{i}, \ldots, \bar{c}_{n}^{i}\right]\right)
\end{aligned}
$$

where $k$ is the kernel size of the convolutional layer. Here we fix $k=3$ as (Ye and Ling 2018).

\footnotetext{
${ }^{1}$ http://nlp.stanford.edu/projects/glove/
}

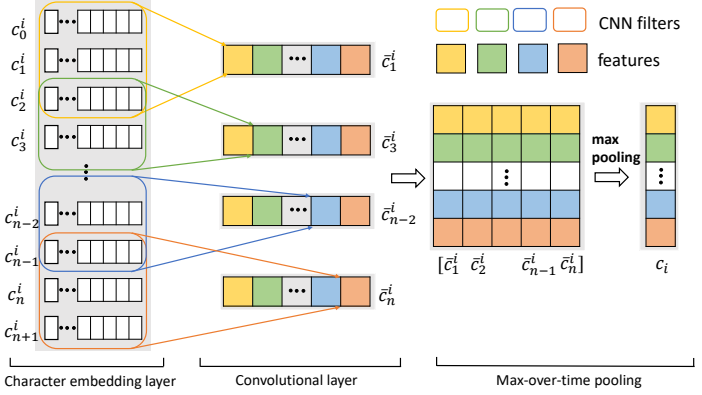

Figure 1: CNN to extract the character-level feature for a word. Best see in color.

Note that RNNs, especially LSTMs/BiLSTMs are also suitable to model the character-level feature. However, as revealed in (Yang, Liang, and Zhang 2018), CNNs are as powerful as RNNs in modelling the character-level feature. Besides, CNNs can probably enjoy higher training and test speed than RNNs. Therefore, in this paper we just adopt a $\mathrm{CNN}$ to model the character-level feature.

We regard $c_{i}$ as the character-level feature for the word $s_{i}$. then we concatenate it to the word-level feature $w_{i}$ to derive the final word feature $z_{i}=\left[c_{i}, w_{i}\right]$.

\section{Context Layer}

Context layer aims to model the local context information among neighboring words for each word. The local context is critical for predicting labels, regarding that there could exist strong dependencies among neighboring words in a sentence. For example, a location word often co-occurs with prepositions like in, on, at. Therefore, it is of necessity to capture the local context information for each word.

And it is obvious that the local dependencies are not limited within a certain distance. Therefore, we should enable the context layer to be adaptive to different scales of local information. Here, like InceptionNet (Szegedy et al. 2015), we design the context layer with different branches, each being comprised of one certain convolutional layer. Figure 2 shows the computational process of the context layer.

Specifically, we use three convolutional layers with the kernel size being $1,3,5$, respectively. After obtaining the word feature $Z=\left\{z_{1}, z_{2}, \ldots, z_{T}\right\}$ of a sentence $s$, each branch firstly extracts the local information $\bar{z}_{i}^{k}$ within a window-size $k$ for each word $s_{i}$. Then a max-pooling operation is employed to select the strongest channel-wise signals from all branches. To add the non-linear characteristic, we also apply tanh after each branch.

$$
\begin{aligned}
& \bar{z}_{i}^{k}=\operatorname{conv}_{k}\left(\left[z_{i-k / 2}, \ldots, z_{i}, \ldots, c_{i+k / 2}\right]\right) \\
& x_{i}=\operatorname{pooling}\left(\left[\tanh \left(\bar{z}_{i}^{1}\right), \tanh \left(\bar{z}_{i}^{3}\right), \tanh \left(\bar{z}_{i}^{5}\right)\right]\right)
\end{aligned}
$$

where $k \in\{1,3,5\}$ is the kernel size. For each $k$, we use $k / 2$ zero-paddings to ensure that each word can get the corresponding context feature. Here, we consider the output $x_{i}$ of the context layer as the context feature for word $s_{i}$.

Although with various kernel sizes, the context layer can capture different kinds of local context information, it still 


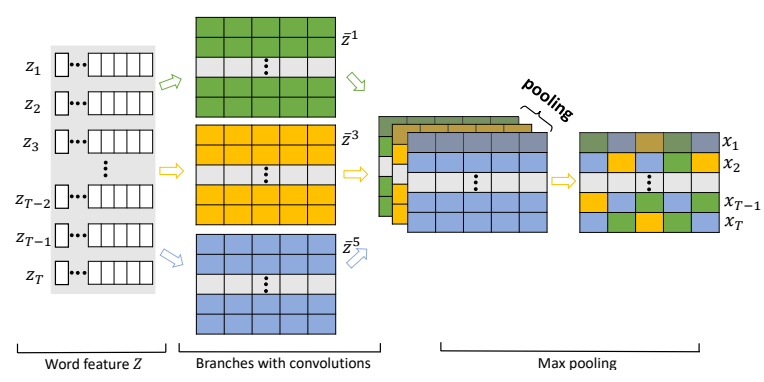

Figure 2: Branches with various convolutions for extracting the local context feature for words. Best see in colors.

struggles to capture the global one. However, we will show that with the gated relation layer described in the following subsection, the global context information can be realized by a fusion of the local one, thus tackling the shortcoming of the context layer.

\section{Relation Layer}

It has been shown that both short-term and long-term context information in a sequence is very critical in sequence learning tasks. LSTMs leverage the memory and the gating mechanism (Hochreiter and Schmidhuber 1997) to capture both context information and gain significant success. However, conventional CNNs cannot well capture the long-term context information owing to the limited receptive fields, and thus existing CNN-based NER models cannot achieve comparable performance as LSTM-based ones.

In this subsection, we introduce the gated relation layer in our proposed GRN, which aims to enhance the conventional CNNs with global context information. Specifically, it models the relations between any two words in the sentence. Then, with the gating mechanism, it composes a global context feature vector by weighted-summing up the relation scores with their corresponding local context feature vectors, as shown in Figure 3. Similar to the attention mechanism, our proposed GRN is effective in modelling long-term dependencies without regard to the limited CNN receptive fields. And importantly, GRN can allow computations to be performed in parallel across the entire sentence, which can generally help to reduce the time costs for training and test.

Given the local context features $\boldsymbol{x}=\left\{x_{1}, x_{2}, \ldots, x_{T}\right\}$ from the context layer for a sentence $s$, the relation layer firstly computes the relation score vector $r_{i j}$ between any two words $s_{i}$ and $s_{j}$, which is of the same dimension as any $x_{i}$. Specifically, it firstly concatenates the corresponding context features $x_{i}$ and $x_{j}$, and then uses a linear function with the weight matrix $W_{r x}$ and the bias vector $b_{r x}$ to obtain $r_{i j}$ :

$$
r_{i j}=W_{r x}\left[x_{i} ; x_{j}\right]+b_{r x}
$$

Like (Santoro et al. 2017), we can directly average these relation score vectors as follows:

$$
r_{i}=\frac{1}{T} \sum_{j=1}^{T} r_{i j}
$$

where $r_{i}$ is the fused global context feature vector for the word $s_{i}$ by the direct feature fusion operation, i.e., averaging in Eq. 5. However, considering that non-entity words generally take up the majority of a sentence, this operation may introduce much noise and mislead the label prediction. To tackle that, we further introduce the gating mechanism, and enable the relation layer to learn to select other dependent words adaptively. Specifically, for the word $s_{i}$, we firstly normalize all its relation score vectors $r_{i j}$ with a sigmoid function to reduce their biases. Then we sum up the normalized relation score vectors $r_{i j}$ with the corresponding local context feature vector $x_{j} \in \boldsymbol{x}=\left\{x_{1}, x_{2}, \ldots, x_{T}\right\}$ of any other word $s_{j}$. And similar to Eq. 5, finally we normalize the sum by the length of the sentence, i.e., $T$.

$$
r_{i}=\frac{1}{T} \sum_{j=1}^{T} \sigma\left(r_{i j}\right) \odot x_{j}
$$

where $\sigma$ is a gate using sigmoid function, and $\odot$ means element-wise multiplication. Note that $r_{i j}$ is asymmetrical and different from $r_{j i}$, and the relation vector w.r.t $s_{i}$ itself, i.e., $r_{i i}$, is also incorporated in the equation above. Therefore, with $r_{i}$ consisting of all the information of other words in the sentence, it can be seen as the global context feature vector for $s_{i}$.

In a way, GRN can be seen as a channel-wise attention mechanism (Chen et al. 2017). However, instead of using a softmax function, we leverage the gating mechanism on the relation score vectors to decide how all the words play a part in predicting the label for the word $s_{i}$. We can also customize Eq. 6 to the formula of attention with gating mechanism, where a gate is used to compute the attention weight for a word:

$$
\begin{aligned}
& \alpha_{i j}=\sigma\left(W_{x}\left[x_{i} ; x_{j}\right]+b_{x}\right) \\
& r_{i}=\frac{1}{T} \sum_{j=1}^{T} \alpha_{i j} * x_{j}
\end{aligned}
$$

where $\alpha_{i j} \in R^{1}$ is an attention weight rather than a vector.

To distinguish from the proposed GRN (i.e., Eq. 6), we name Eq. 5 as Direct Fusion Network (DFN) and Eq. 7 as Gated Attention Network (GAttN). We will consider DFN and GAttN as two of our baseline models to show the superiority of the proposed GRN.

Here we also add a non-linear function for $r_{i}$ as follows.

$$
p_{i}=\tanh \left(r_{i}\right)
$$

And we define $p_{i}$ as the final predicting feature for word $s_{i}$.

\section{CRF Layer}

Modelling label dependencies is crucial for NER task (Ma and Hovy 2016; Liu et al. 2018). Following (Ma and Hovy 2016; Huang, $\mathrm{Xu}$, and Yu 2015), we employ a conditional random field (CRF) layer to model the label dependencies and calculate the loss for training GRN.

Formally, for a given sentence $s=\left\{s_{1}, s_{2}, \ldots, s_{T}\right\}$ and its generic sequence of labels $\boldsymbol{y}=\left\{y_{1}, y_{2}, \ldots, y_{T}\right\}$, we firstly use $\mathcal{Y}(s)$ to denote the set of all possible label sequences for 


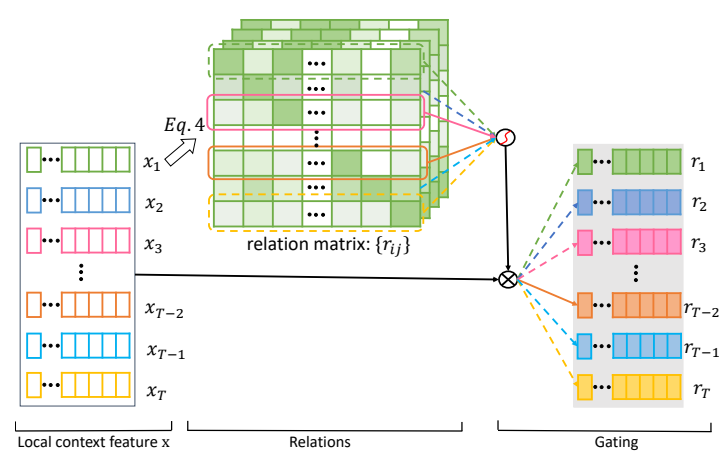

Figure 3: Gated relation layer in GRN for composing the global context feature for each word. $r_{i j}$ denotes the relation score vector between word $s_{i}$ and word $s_{j}$. Best see in color.

$s$. The CRF model defines a family of conditional probability $p(\boldsymbol{y} \mid \boldsymbol{s})$ over all possible label sequences $\boldsymbol{y}$ given $\boldsymbol{s}$ :

$$
p(\boldsymbol{y} \mid \boldsymbol{s})=\frac{\prod_{i=1}^{T} \phi_{i}\left(y_{i-1}, y_{i}, \boldsymbol{s}\right)}{\sum_{y^{\prime} \in \mathcal{Y}(\boldsymbol{s})} \prod_{i=1}^{T} \phi_{i}\left(y_{i-1}^{\prime}, y_{i}^{\prime}, \boldsymbol{s}\right)}
$$

where $\phi_{i}\left(y_{i-1}, y_{i}, s\right)=\exp \left(f\left(s_{i}, y^{\prime}, y\right)\right)$ with $f$ being a function that maps words into labels:

$$
f\left(s_{i}, y^{\prime}, y\right)=W_{y} p_{i}+b_{y^{\prime}, y}
$$

where $p_{i}$ is derived as Eq. 8, $W_{y}$ is the predicting weights w.r.t $y$ and $b_{y^{\prime}, y}$ is the transition weight from $y^{\prime}$ to $y$. Both $W_{y}$ and $b_{y^{\prime}, y}$ are parameters to be learned.

Loss of the CRF layer is formulated as follows.

$$
L=-\sum_{\boldsymbol{s}} \log p(\boldsymbol{y} \mid \boldsymbol{s})
$$

And for decoding, we aim to find the label sequence $\boldsymbol{y} *$ with the highest conditional probability:

$$
\boldsymbol{y} *=\arg \max _{\boldsymbol{y} \in \mathcal{Y}(\boldsymbol{s})} p(\boldsymbol{y} \mid \boldsymbol{s})
$$

which can be efficiently derived via Viterbi decoding.

\section{Experiments}

To verify the effectiveness of the proposed GRN, we conduct extensive experiments on two benchmark NER datasets: CoNLL-2003 English NER (Tjong Kim Sang and De Meulder 2003) and OntoNotes 5.0 (Hovy et al. 2006; Pradhan et al. 2013).

- CoNLL-2003 English NER consists of 22,137 sentences totally and is split into 14,987, 3,466 and 3,684 sentences for the training set, the development set and the test set, respectively. It includes annotations for 4 types of named entities: PERSON, LOCATION, ORGANIZATION and MISC.

- OntoNotes $\mathbf{5 . 0}$ consists of much more $(76,714)$ sentences from a wide variety of sources (telephone conversation, newswire, etc.). Following (Chiu and Nichols 2016), we use the portion of the dataset with gold-standard named entity annotations, and thus excluded the New Testaments portion. It also contains much more kinds of entities, including CARDINAL, MONEY, LOC, PRODUCT, etc.

\begin{tabular}{|l|c|c|c|c|}
\hline dataset & & Train & Dev & Test \\
\hline \multirow{3}{*}{ CoNLL-2003 } & Sentence & 14,987 & 3,466 & 3,684 \\
& Token & 204,567 & 51,578 & 46,666 \\
& Entity & 23,499 & 5,942 & 5,648 \\
\hline \multirow{3}{*}{ OntoNotes 5.0 } & Sentence & 59,924 & 8,528 & 8,262 \\
& Token & $1,088,503$ & 147,724 & 152,728 \\
& Entity & 81,828 & 11,066 & 11,257 \\
\hline
\end{tabular}

Table 1: Statistics of CoNLL-2003 and Ontonotes 5.0.

Table 1 shows some statistics of both datasets. Following (Ma and Hovy 2016), we use the BIOES sequence labelling scheme instead of BIO for both datasets to train models. As for test, we convert the prediction results back to the BIO scheme and use the standard CoNLL-2003 evaluation script to measure the NER performance, i.e., $F_{1}$ scores, etc.

\section{Network Training}

We implement our proposed GRN with the Pytorch library (Paszke et al. 2017). And we set the parameters below following (Ma and Hovy 2016).

Word Embeddings. The dimension of word embedding is set as 100. And as mentioned, we initialize it with Stanford's publicly available GloVe 100-dimensional embeddings. We include all words of GloVe when building the vocabulary, besides those words appearing at least 3 times in the training set. For words out of the vocabulary (denoted as UNK) or those not in GloVe, we initialize their embeddings with kaiming uniform initialization (He et al. 2015).

Character Embeddings. We set the dimension of character embeddings as 30 , and also initialize them with kaiming uniform initialization.

Weight Matrices and Bias Vectors. All weight matrices in linear functions and CNNs are initialized with kaiming uniform initialization, while bias vectors are initialized as 0 .

Optimization. We employ mini-batch stochastic gradient descent with momentum to train the model. The batch size is set as 10 . The momentum is set as 0.9 and the initial learning rate is set as 0.02 . We use learning rate decay strategy to update the learning rate during training. Namely, we update the learning rate as $\frac{0.02}{1+\rho * t}$ at the $t$-th epoch with $\rho=0.02$. We train each model on training sets with 200 epochs totally, using dropout $=0.5$. For evaluation, we select its best version with the highest performance on the development set and report the corresponding performance on the test set. To reduce the model bias, we carry out 5 runs for each model and report the average performance and the standard deviation.

Network Structure. The output channel number of the CNN in Eq. 2 and Eq. 3 is set as 30 and 400, respectively.

\section{Performance Comparison}

Here we first focus on the NER performance comparison between the proposed GRN and the existing state-of-the-art approaches.

CoNLL-2003. We compare GRN with various state-ofthe-art LSTM-based NER models, including (Liu et al. 2018; Ye and Ling 2018), etc. We also compare GRN with ID-CNN (Strubell et al. 2017), which also adopts CNNs without recurrent layers for NER. Furthermore, considering that some state-of-the-art NER models exploit external 


\begin{tabular}{|l|c|c|c|}
\hline Model & Mean $( \pm s t d) \mathrm{F}_{1}$ & Max F $_{1}$ & Mean P/R \\
\hline (Collobert et al. 2011) & 88.67 & & \\
\hline (Luo et al. 2015) & 89.9 & & $90.0 / 89.9$ \\
\hline (Chiu and Nichols 2016) & $90.91 \pm 0.20$ & & $90.75 / 91.08$ \\
\hline (Zhuo et al. 2016) & 88.12 & & \\
\hline (Rei, Crichton, and Pyysalo 2016) & 84.09 & & \\
\hline (Lample et al. 2016) & 90.94 & & \\
\hline (Ma and Hovy 2016) & 91.21 & & $\mathbf{9 1 . 3 5} / 91.06$ \\
\hline (Rei 2017) & 86.26 & & \\
\hline (Zukov-Gregoric et al. 2017) & 89.83 & & \\
\hline (Liu, Baldwin, and Cohn 2017) & 89.5 & & \\
\hline (Peters et al. 2017) & $90.87 \pm 0.13$ & & \\
\hline (Liu et al. 2018) & $91.24 \pm 0.12$ & 91.35 & \\
\hline (Ye and Ling 2018) & $91.38 \pm 0.10$ & 91.53 & \\
\hline ID-CNN (Strubell et al. 2017) & $90.54 \pm 0.18$ & & \\
\hline CNN-BiLSTM-CRF & $91.17 \pm 0.18$ & 91.45 & $90.98 / 91.35$ \\
\hline GRN & $\mathbf{9 1 . 4 4} \pm \mathbf{0 . 1 6}$ & $\mathbf{9 1 . 6 7}$ & $91.31 / \mathbf{9 1 . 5 7}$ \\
\hline \hline (Collobert et al. 2011)* $^{*}$ & 89.59 & & \\
\hline Luo et al. 2015)* $^{*}$ & 91.2 & & $91.5 / 91.4$ \\
\hline (Chiu and Nichols 2016)* & $91.62 \pm 0.33$ & & $91.39 / 91.85$ \\
\hline (Peters et al. 2017)* $^{*}$ & $91.93 \pm 0.19$ & & \\
\hline (Tran, MacKinlay, and Yepes 2017)* & 91.66 & & \\
\hline (Yang, Salakhutdinov, and Cohen 2017)* & 91.26 & & \\
\hline (Peters et al. 2018)* $^{*}$ & $92.22 \pm 0.10$ & & \\
\hline GRN* & $\mathbf{9 2 . 3 4} \pm \mathbf{0 . 1 0}$ & $\mathbf{9 2 . 4 5}$ & $\mathbf{9 2 . 0 4 / 9 2 . 6 5}$ \\
\hline & & & \\
\hline
\end{tabular}

Table 2: Performance comparison on CoNLL-2003. * indicates models utilizing external knowledge besides the CoNLL-2003 training set and pre-trained word embeddings. $\mathrm{P} / \mathrm{R}$ denotes precision and recall.

knowledge to boost their performance, here we also report the performance of GRN with ELMo (Peters et al. 2018) incorporated as the external knowledge. Note that ELMo is trained on a large corpus of text data and can generate $d y$ namic contextual features for words in a sentence. Here we simply concatenate the output ELMo features to the word feature in GRN. The experimental results are reported in Table 2, which also includes the max $F_{1}$ scores, mean precision and recall values if available. Note that CNN-BiLSTM-CRF is our re-implementation of (Ma and Hovy 2016), and we obtain comparable performance as that reported in the paper. Therefore, by default we directly compare GRN with the reported performance of compared baselines. It should also be noticed that, since the relation layer in GRN can be related to the attention mechanism, here we also include some attention-based baselines, i.e.,, (Rei, Crichton, and Pyysalo 2016) and (Zukov-Gregoric et al. 2017).

As shown in Table 2, compared with those LSTM-based NER models, the proposed GRN can obtain comparable or even slightly superior performance, with or without the external knowledge, which well demonstrates the effectiveness of GRN. And compared with ID-CNN, our proposed GRN can defeat it at a great margin in terms of $\mathrm{F}_{1}$ score. We also try to add ELMo to the latest state-of-the-art model of (Ye and Ling 2018) based on their published codes, and we find that the corresponding $\mathrm{F}_{1}$ score is $91.79 \pm 0.08$, which is substantially lower than that of GRN.

OntoNotes 5.0. On OntoNotes 5.0, we compare the proposed GRN with NER models that also reported performance on it, including (Chiu and Nichols 2016; Shen et al. 2017; Durrett and Klein 2014), etc. As shown in Table 3, GRN can obtain the state-of-the-art NER performance on OntoNotes 5.0, which further demonstrates its effectiveness.

Overall, the comparison results on both CoNLL-2003 and OntoNotes 5.0 well indicate that our proposed GRN can

\begin{tabular}{|l|c|c|}
\hline Model & Mean $( \pm s t d) \mathrm{F}_{1}$ & Mean P/R \\
\hline (Passos, Kumar, and McCallum 2014) & 82.30 & \\
\hline (Durrett and Klein 2014) & 84.04 & $85.22 / 82.89$ \\
\hline (Chiu and Nichols 2016) & $86.28 \pm 0.26$ & $86.04 / 86.53$ \\
\hline (Shen et al. 2017) & $86.63 \pm 0.49$ & \\
\hline ID-CNN (Strubell et al. 2017) & $86.84 \pm 0.19$ & \\
\hline GRN & $\mathbf{8 7 . 6 7} \pm \mathbf{0 . 1 7}$ & $\mathbf{8 7 . 7 9} / \mathbf{8 7 . 5 6}$ \\
\hline
\end{tabular}

Table 3: Performance comparison on OntoNotes 5.0. P/R denotes precision and recall.

\begin{tabular}{|l|l|c|c|}
\hline & Model & Mean $( \pm$ std $) \mathrm{F}_{1}$ & $\mathrm{~F}_{1}$ Drop \\
\hline \multirow{2}{*}{ context } & GRN w/o context & $88.36 \pm 0.21$ & 3.08 \\
\cline { 2 - 4 } & GRN w/branch & $90.88 \pm 0.22$ & 0.56 \\
\hline \hline \multirow{3}{*}{ relation } & GRN w/o relation & $90.13 \pm 0.28$ & 1.31 \\
\cline { 2 - 4 } & DFN & $90.72 \pm 0.06$ & 0.72 \\
\cline { 2 - 4 } & GAttN & $87.11 \pm 0.25$ & 4.33 \\
\hline \hline Full & GRN & $\mathbf{9 1 . 4 4} \pm \mathbf{0 . 1 6}$ & 0 \\
\hline
\end{tabular}

Table 4: Ablation study on CoNLL-2003.

\begin{tabular}{|l|l|c|c|}
\hline & Model & Mean $( \pm$ std $) \mathrm{F}_{1}$ & $\mathrm{~F}_{1}$ Drop \\
\hline \multirow{2}{*}{ context } & GRN w/o context & $82.21 \pm 0.23$ & 5.46 \\
\cline { 2 - 4 } & GRN w/ branch & \\
3 & $86.66 \pm 0.21$ & 1.01 \\
\hline \hline \multirow{3}{*}{ relation } & GRN w/o relation & $85.87 \pm 0.16$ & 1.80 \\
\cline { 2 - 4 } & DFN & $85.81 \pm 0.14$ & 1.86 \\
\cline { 2 - 4 } & GAttN & $79.83 \pm 0.37$ & 7.83 \\
\hline \hline Full & GRN & $\mathbf{8 7 . 6 7} \pm \mathbf{0 . 1 7}$ & 0 \\
\hline
\end{tabular}

Table 5: Ablation study on OntoNotes 5.0.

achieve state-of-the-art NER performance with or without external knowledge. It demonstrates that, using GRN, CNNbased models can compete with LSTM-based ones for NER.

\section{Ablation Study}

Here we study the impact of each layer on GRN. Firstly, we analyze the context layer by introducing two baseline models: (1) GRN w/o context: wiping out the context layer and building the relation layer on top of the representation layer directly; (2) GRN w/ branch 3 : removing branches in the context layer, except the one with kernel size $=3$. Then to analyze the relation layer and the importance of gating mechanism in it, we compare GRN with: (1) GRN w/o relation: wiping out the relation layer and directly building the CRF layer on top of the context feature; (2) DFN (see Eq. 5); (3) GAttN (see Eq. 7). All compared baselines use the same experimental settings as GRN. Table 4 and Table 5 report the experimental results on both datasets, where the last column shows the absolute performance drops compared to GRN.

As shown in Table 4 and Table 5, when reducing the number of branches in the context layer, GRN w/o context and GRN w/ branch ${ }_{3}$ drop significantly, which indicates that modelling different scales of local context information plays a crucial role for NER.

Compared with GRN w/o relation, DFN and GAttN, the proposed GRN defeats them at a substantial margin in terms of $\mathrm{F}_{1}$ score, which demonstrates that the proposed gated relation layer is beneficial to the performance improvement. The comparison also reveals that the channel-wise gating mechanism in GRN is more powerful than the gated atten- 


\begin{tabular}{|l|c|c|}
\hline & CoNLL-2003 & OntoNotes 5.0 \\
\hline Training & $1.16 \mathrm{x}$ & $1.15 \mathrm{x}$ \\
\hline Test & $1.19 \mathrm{x}$ & $1.08 \mathrm{x}$ \\
\hline
\end{tabular}

Table 6: Training/test speedup of GRN compared with CNN-BiLSTM-CRF.

tion approach (i.e., Eq. 7) and the direct fusion approach (i.e., Eq. 5) under the same experimental settings for NER.

\section{Training/Test Time Comparison}

In this section, we further compare the training and test time costs of the proposed GRN with those of CNN-BiLSTMCRF, which is the most basic LSTM-based NER model achieving high performance. We conduct our experiments on a physical machine with Ubuntu 16.04, 2 Intel Xeon E52690 v4 CPUs, and a Tesla P100 GPU. For fair comparison, we keep the representation layer and the CRF layer the same for both models, so that the input and output dimensions for the "BiLSTM layer" in CNN-BiLSTM-CRF would be identical to those of the "context layer + relation layer" in GRN. We train both models with random initialization for a total of 30 epochs, and after each epoch, we evaluate the learned model on the test set. For both training and test, batch size is set as 10 as before. And here we use the average training time per epoch and the average test time to calculate speedups.

As shown in Table 6, GRN can obtain a speedup of more than 1.15 during training and around 1.10 during test on both datasets. The speedup may seem not so significant, because the time costs reported here also include those consumed by common representation layer, CRF layer, etc. For reference, the fast ID-CNN with a CRF layer (i.e., ID-CNN-CRF) (Strubell et al. 2017) was reported to have a test-time speedup of 1.28 over the basic BiLSTM-CRF model on CoNLL-2003. Compared to ID-CNN-CRF, GRN sacrifices some speedup for better performance, and the speedup gap between both is still reasonable. We can also see that the speedup on CoNLL-2003 is larger than that on OntoNotes 5.0, which can be attributed to that the average sentence length of CoNLL-2003 $(\sim 14)$ is smaller than that of OntoNotes $5.0(\sim 18)$ and thus the relation layer in GRN would cost less time for the former. The results above demonstrate that the proposed GRN can generally bring efficiency improvement over LSTM-based methods for NER, via fully exploiting the GPU parallelism.

\section{Word Relation Visualization}

Since the proposed GRN aims to boost the NER performance by modelling the relations between words, especially long-term ones, we can visualize the gating output in the relation layer to illustrate the interpretability of GRN. Specifically, we utilize the $L 2$ norm of $r_{i j}$ to indicate the extent of relations between the word $s_{i}$ and the word $s_{j}$. Then we further normalize the values into $[0,1]$ to build a heat map. Figure 4 shows a visualization sample. We can find out that the entity words (y-axis) are more related to other entity words as well, even though they may be "far away" from each other

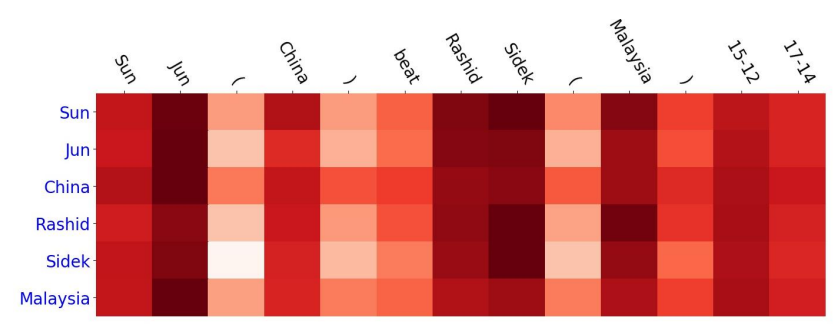

Figure 4: Word relation visualization: the $\mathrm{x}$-axis shows the sentence and the y-axis shows the entity words in it. Regions with deeper color means stronger relations between the corresponding pair of words.

in the sentence, like the 1st word "Sun" and the 8th word "Sidek" in the sample. Note that "Sun" and "Sidek" are not in an identical receptive field of any CNN used in our experiments, but their strong correlation can still be exploited with the relation layer in GRN. That concretely illustrates that, by introducing the gated relation layer, GRN is able to capture the long-term dependency between words.

\section{Conclusion}

In this paper, we propose a CNN-based network, i.e., gated relation network (GRN), for named entity recognition (NER). Unlike the dominant LSTM-based NER models which process a sentence in a sequential manner, GRN can process all the words concurrently with one forward operation and thus can fully exploit the GPU parallelism for potential efficiency improvement. Besides, compared with common CNNs, GRN has a better capacity of capturing long-term context information. Specifically, GRN introduces a gated relation layer to model the relations between any two words, and utilizes gating mechanism to fuse local context features into global ones for all words. Experiments on CoNLL-2003 English NER and Ontonotes 5.0 datasets show that, GRN can achieve state-of-the-art NER performance with or without external knowledge, meaning that using GRN, CNN-based models can compete with LSTMbased models for NER. Experimental results also show that GRN can generally bring efficiency improvement for training and test.

\section{Acknowledgements}

This work is jointly supported by the National Natural Science Foundation of China (No. 61571269) and the National Key R\&D Program of China (No. 2018YFC0807500).

\section{References}

Bikel, D. M.; Miller, S.; Schwartz, R.; and Weischedel, R. 1997. Nymble: a high-performance learning name-finder. In ANLP, 194-201.

Chen, L.; Zhang, H.; Xiao, J.; Nie, L.; Shao, J.; Liu, W.; and Chua, T.-S. 2017. Sca-cnn: Spatial and channel-wise attention in convolutional networks for image captioning. In CVPR, 6298-6306. 
Chiu, J., and Nichols, E. 2016. Named entity recognition with bidirectional lstm-cnns. TACL 4(1):357-370.

Collobert, R.; Weston, J.; Bottou, L.; Karlen, M.; Kavukcuoglu, K.; and Kuksa, P. 2011. Natural language processing (almost) from scratch. JMLR 12:2493-2537.

Durrett, G., and Klein, D. 2014. A joint model for entity analysis: Coreference, typing, and linking. TACL 2(1):477490.

Gehring, J.; Auli, M.; Grangier, D.; Yarats, D.; and Dauphin, Y. N. 2017. Convolutional sequence to sequence learning. In ICML, 1243-1252.

He, K.; Zhang, X.; Ren, S.; and Sun, J. 2015. Delving deep into rectifiers: Surpassing human-level performance on imagenet classification. In ICCV, 1026-1034.

Hochreiter, S., and Schmidhuber, J. 1997. Long short-term memory. Neural Computation 9(8):1735-1780.

Hovy, E.; Marcus, M.; Palmer, M.; Ramshaw, L.; and Weischedel, R. 2006. Ontonotes: the $90 \%$ solution. In NAACL-HLT, 57-60.

Huang, Z.; Xu, W.; and Yu, K. 2015. Bidirectional lstm-crf models for sequence tagging. arXiv:1508.01991.

Lample, G.; Ballesteros, M.; Subramanian, S.; Kawakami, K.; and Dyer, C. 2016. Neural architectures for named entity recognition. In NAACL-HLT, 260-270.

Liu, F.; Baldwin, T.; and Cohn, T. 2017. Capturing longrange contextual dependencies with memory-enhanced conditional random fields. In IJCNLP, 555-565.

Liu, L.; Shang, J.; Xu, F.; Ren, X.; Gui, H.; Peng, J.; and Han, J. 2018. Empower Sequence Labeling with TaskAware Neural Language Model. In AAAI.

Luo, G.; Huang, X.; Lin, C.-Y.; and Nie, Z. 2015. Joint entity recognition and disambiguation. In EMNLP, 879-888.

Ma, X., and Hovy, E. 2016. End-to-end sequence labeling via bi-directional lstm-cnns-crf. In ACL, 1064-1074.

McCallum, A., and Li, W. 2003. Early results for named entity recognition with conditional random fields, feature induction and web-enhanced lexicons. In CoNLL, 188-191.

Passos, A.; Kumar, V.; and McCallum, A. 2014. Lexicon infused phrase embeddings for named entity resolution. In CoNLL, 78-86.

Paszke, A.; Gross, S.; Chintala, S.; Chanan, G.; Yang, E.; DeVito, Z.; Lin, Z.; Desmaison, A.; Antiga, L.; and Lerer, A. 2017. Automatic differentiation in pytorch. In NIPS.

Pennington, J.; Socher, R.; and Manning, C. 2014. Glove: Global vectors for word representation. In EMNLP, 15321543.

Peters, M.; Ammar, W.; Bhagavatula, C.; and Power, R. 2017. Semi-supervised sequence tagging with bidirectional language models. In ACL, 1756-1765.

Peters, M.; Neumann, M.; Iyyer, M.; Gardner, M.; Clark, C.; Lee, K.; and Zettlemoyer, L. 2018. Deep contextualized word representations. In NAACL-HLT, 2227-2237.

Pradhan, S.; Moschitti, A.; Xue, N.; Ng, H. T.; Björkelund, A.; Uryupina, O.; Zhang, Y.; and Zhong, Z. 2013. Towards robust linguistic analysis using ontonotes. In CoNLL, 143152.

Rei, M.; Crichton, G.; and Pyysalo, S. 2016. Attending to characters in neural sequence labeling models. In COLING, 309-318.

Rei, M. 2017. Semi-supervised multitask learning for sequence labeling. In $A C L, 2121-2130$.

Santoro, A.; Raposo, D.; Barrett, D. G.; Malinowski, M.; Pascanu, R.; Battaglia, P.; and Lillicrap, T. 2017. A simple neural network module for relational reasoning. In NIPS, 4967-4976.

Shen, Y.; Yun, H.; Lipton, Z.; Kronrod, Y.; and Anandkumar, A. 2017. Deep active learning for named entity recognition. In Workshop on Representation Learning for NLP, 252-256.

Strubell, E.; Verga, P.; Belanger, D.; and McCallum, A. 2017. Fast and accurate entity recognition with iterated dilated convolutions. In EMNLP, 2670-2680.

Szegedy, C.; Liu, W.; Jia, Y.; Sermanet, P.; Reed, S.; Anguelov, D.; Erhan, D.; Vanhoucke, V.; and Rabinovich, A. 2015. Going deeper with convolutions. In CVPR, 1-9.

Tjong Kim Sang, E. F., and De Meulder, F. 2003. Introduction to the conll-2003 shared task: Language-independent named entity recognition. In CoNLL, 142-147.

Tran, Q.; MacKinlay, A.; and Yepes, A. J. 2017. Named entity recognition with stack residual 1 stm and trainable bias decoding. In IJCNLP, 566-575.

Vaswani, A.; Shazeer, N.; Parmar, N.; Uszkoreit, J.; Jones, L.; Gomez, A. N.; Kaiser, Ł.; and Polosukhin, I. 2017. Attention is all you need. In NIPS, 5998-6008.

Yang, J.; Liang, S.; and Zhang, Y. 2018. Design challenges and misconceptions in neural sequence labeling. In $C O L$ ING, 3879-3889.

Yang, Z.; Salakhutdinov, R.; and Cohen, W. W. 2017. Transfer learning for sequence tagging with hierarchical recurrent networks. arXiv:1703.06345.

Ye, Z., and Ling, Z.-H. 2018. Hybrid semi-markov crf for neural sequence labeling. In $A C L, 235-240$.

Zhuo, J.; Cao, Y.; Zhu, J.; Zhang, B.; and Nie, Z. 2016. Segment-level sequence modeling using gated recursive semi-markov conditional random fields. In ACL, 14131423.

Zukov-Gregoric, A.; Bachrach, Y.; Minkovsky, P.; Coope, S.; and Maksak, B. 2017. Neural named entity recognition using a self-attention mechanism. In ICTAI, 652-656. 